\title{
High-sensitivity microwave vector detection at extremely low-power levels for low-dimensional electron systems
}

\author{
W. H. Hsieh and C. H. Kuan \\ Graduate Institute of Electronics Engineering and Department of Electrical Engineering, \\ National Taiwan University, Taipei, Taiwan, Republic of China \\ Y. W. Suen, ${ }^{\text {a) }}$ S. Y. Chang, and L. C. Li \\ Department of Physics, National Chung Hsing University, 250, \\ Kuo Kuang Road, Taichung 402, Taiwan, Republic of China \\ B. C. Lee and C. P. Lee \\ Department of Electronics Engineering, National Chiao Tung University, Sinchu, Taiwan, Republic of China
}

(Dated: March 20, 2021)

\begin{abstract}
We present a high-sensitivity microwave vector detection system for studying the low-dimensional electron system embedded in the gaps of a coplanar waveguide at low temperatures. Using this system, we have achieved $0.005 \%$ and $0.001^{\circ}$ resolutions in amplitude and phase variations, respectively, at $10 \mathrm{GHz}$ in a magnetotransport measurement on a quantum-wire array with an average signal power less than $-75 \mathrm{dBm}$ into the sample at $0.3 \mathrm{~K}$. From the measured phase variation, we can distinguish a very tiny change in the induced dipole moment of each quantum wire.
\end{abstract}

Coplanar waveguides (CPW's) have been successfully used as broadband sensors in investigating the high-frequency magnetotransport phenomena of lowdimensional electron systems (LDES's), such as twodimensional electron systems (2DES's) $\stackrel{1,2,3.4,5}{,}$ and anti quantum dots (QD's),$\frac{6}{,}$ etc. In these works, a commercial vector network analyzer (VNA) is the major tool to measure the variation of the propagation constant, including the attenuation constant $(\alpha)$ and the phase constant $(\beta)$, of the CPW that containing the active LDES in the gaps between the metal electrodes. From $\alpha$ and $\beta$ one can extract the longitudinal conductivity $\left(\sigma_{x x}\right)^{\frac{1}{\underline{1}}}$ (both real and imaginary parts) of the LDES. However, since the microwave power delivered to samples at temperature $(T)$ below few hundred mK must be very low, the resolution of the data becomes very poor, especially for the phase part. Thus in most of the previous studies using CPW sensors, they only presented $\operatorname{Re}\left\{\sigma_{x x}\right\}$ data derived from $\alpha$ and discarded the phase part. Even though Hohls et $a l^{7}$ and Lewis et al $l^{8}$ have addressed the $\operatorname{Im}\left\{\sigma_{x x}\right\}$ behavior in the integer quantum Hall (IQH) regime based on other techniques, still, the resolution of $\operatorname{Im}\left\{\sigma_{x x}\right\}$ is mediocre due to the constrain of VNA's. Nevertheless, $\operatorname{Im}\left\{\sigma_{x x}\right\}$, proportional to the change of the real part of dielectric constant, gives important information about the electric polarization, that is of special interest in the case of QD's, quantum wires (QW's), or 2DES's at high magnetic fields $(B)$. Furthermore, the relatively small effective area of QD's or QW's compared to 2DES samples leads to a very small signal variation (or dynamic range), that makes the conventional VNA measurement very difficult and impractical.

\footnotetext{
a) Author to whom correspondence should be addressed; electronic mail: ysuen@phys.nchu.edu.tw
}

In this letter, we present a new detection scheme and the instrumental implementation, which can resolve very small variations not only in the amplitude but also the phase of an extremely low-power-level microwave signal traveling through a CPW with LDES's embedded in the gaps while some external sample parameters, such as the applied magnetic field $(B)$ or $T$, etc., is changed. The data of a low- $T$ magnetotransport measurement on a QW-array sample manifest the high-resolution capability of this system.

A simplified schematic diagram to illustrate the principle of phase detection by a phase-lock loop ${ }^{9}$ (PLL) is depicted in Fig. 1 (a). The CPW sample is connected to a PLL through two semirigid coaxial cables of total length $L$. The PLL will force the total phase change $(\Delta \phi)$, including the phase change of the semirigid cables $\left(\Delta \phi_{L}\right)$ and the CPW sample $\left(\Delta \phi_{s}\right)$, to be 0 by tuning the frequency $(f)$ of the voltage-controlled oscillator (VCO) during the experiments, that is, $\Delta \phi=\Delta \phi_{L}+\Delta \phi_{s}=0$, or $\Delta \phi_{s}=-\Delta \phi_{L}$. Hence $\Delta \phi_{s}$ can be obtained directly from the frequency change $(\Delta f)$ of $\mathrm{VCO}$ via

$$
\Delta \phi_{s}=-\Delta \phi_{L}=-2 \pi \Delta f L / v_{L}=-\Delta \omega \tau_{L}
$$

where $v_{L}$ is the phase velocity of the signal in the cable. The result can be expressed as the product of the change of the angular frequency $(\Delta \omega)$ and the delay time $\left(\tau_{L}\right)$ of the connecting cables with a different sign.

A complete block diagram, including the pulse handling circuits, the microwave PLL, and the amplitude readout circuit, together with the CPW sample in a cryogenic environment, is shown in Fig. 1 (b). The microwave part of this system is basically a pair of homodyne detectors (mixers) with reference signals of quadrature phase difference. One of the mixers with $0^{\circ}$ reference $(L O 1)$, used as the phase sensitive detector (PSD), has zero output (IF1) forced by the PLL, while the other one with 


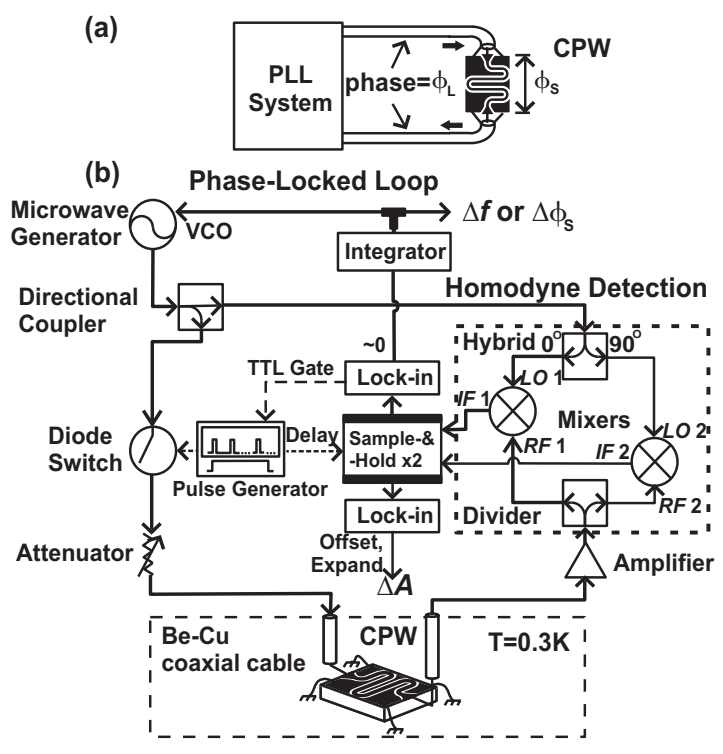

FIG. 1: (a) Simplified schematic diagram for phase detection using a PLL. (b) Block diagram of the vector detection system. The meandering CPW containing a LDES in the gaps is part of the microwave signal path in the PLL.

$90^{\circ}$ reference ( $L O 2$ ) has an output (IF2) proportional to the amplitude of the signal.

Besides homodyne detection we employ a double-pulse modulation scheme to detect and average the microwave signal. A short pulse train with a $0.2 \sim 2 \mu$ s pulse width and a $0.1 \sim 10 \%$ duty cycle, provided by a pulse generator and gated by a slow square-wave TTL signal with a period of $1 \sim 10 \mathrm{~ms}$ from a lock-in amplifier, modulates the microwave signal sent to the sample. A time-delayed pulse with a $0.1 \sim 1 \mu$ s pulse width triggered by the modulating pulses controls a sample-and-hold $(\mathrm{S} \& \mathrm{H})$ circuit that samples the IF output of the microwave mixer. The holding capacitor in the $\mathrm{S} \& \mathrm{H}$ circuit is discharged through an analog switch when the TTL gating signal is low. Finally the lock-in amplifier reads the output of the $\mathrm{S} \& \mathrm{H}$ circuit. There are two sets of pulse averaging circuits, one for the PLL and the other for the amplitude readout. Note that the time constant of the lock-in amplifier for the PLL is about $1 \sim 30 \mathrm{~ms}$, in contrast to 300 $\mathrm{ms}$ or $1 \mathrm{~s}$ for the amplitude readout part. The average of the PSD output (IF1) is sent to an integrator (loop filter of PLL) with a time constant of $26.3 \mathrm{~ms}$. The output of the integrator connects to the FM input of the microwave source (VCO), thus closing the PLL. In fact this PLL system is modified from what people used in surface-acoustic-wave detection experiments $\stackrel{10}{\underline{\underline{10}}}$ but with improved pulse averaging and amplitude detection methods.

We use three sets of microwave modules to cover the frequency from about $60 \mathrm{MHz}$ to $18 \mathrm{GHz}$. The details of our instrumentations and circuit designs will be published elsewhere. To gain an idea of the detection limit, we tested our system with only an $11 \mathrm{~m}$ semirigid ca- ble connected to the PLL without samples. The input microwave signal is attenuated down to $-70 \mathrm{dBm}$ peak power, and less than $-90 \mathrm{dBm}$ in average. The background phase fluctuation we obtain in this test is less than $0.0003^{\circ}$ (root-mean-squared value) for $f \lesssim 6 \mathrm{GHz}$ and $0.006^{\circ}$ for $6 \lesssim f \lesssim 18 \mathrm{GHz}$, which is remarkably low for such a low-power signal. In fact, the signal power reaching the low-noise amplifier (LNA) is even lower than the input value claimed above due to the loss of the cable, which is about $-9 \mathrm{~dB}$ at $1 \mathrm{GHz}$ and raises to $-41 \mathrm{~dB}$ at 10 GHz. This may explain why the noise in phase increases at high frequencies. The resolution with a low- $T$ sample loaded is slightly worse due to the loss of the sample and extra noise from the cryogenic environment. The resolution of the amplitude readout for a small-variation signal can be enhanced by the use of the "offset" and "expand" functions of the lock-in amplifier ${ }^{11}$

In the following we will present measured results for a QW array sample to demonstrate the resolving power of this method. The sample is fabricated from a standard MBE-grown modulation-doped GaAs/AlGaAs heterostructure containing a 2DES, which is $150 \mathrm{~nm}$ under the surface. The 2 DES has a mobility of about $1.5 \times 10^{5}$ $\mathrm{cm}^{2} / \mathrm{Vs}$ at $4 \mathrm{~K}$, and a density of $1.1 \times 10^{11} \mathrm{~cm}^{-2}$. Before evaporating the $\mathrm{Cr} / \mathrm{Au}(10 / 300 \mathrm{~nm})$ metal layers for the CPW pattern, we etch away the 2DES part underneath. The widths of the center conductor and the gap of the $50 \Omega \mathrm{CPW}$ are $36 \mu \mathrm{m}$ and $23 \mu \mathrm{m}$, respectively. A meandering pattern 1 is used to increase the effective length of the CPW. Subsequently we pattern the 2DES left in the gap into about 7000 identical QW mesas, each of 0.7 $\mu \mathrm{m}$ wide and $20 \mu \mathrm{m}$ long, by using e-beam lithography and chemical etching [Fig. 2 (a)]. The QW's, parallel to the propagating direction of microwave signals, occupy only about $6 \mathrm{~mm}$ in length of the straight sections of the meandering $\mathrm{CPW}$.

The CPW sample is immersed in liquid ${ }^{3} \mathrm{He}(0.3 \mathrm{~K})$ with applied $B$ perpendicular to the sample surface. The total time delay given by the connection cables and microwave modules is $51.1 \mathrm{~ns}$. From Eq. 1, this time delay multiplied by $\Delta f$ gives the phase change $\left(\Delta \phi_{s}\right)$ of the CPW sample. Here $\Delta f$ can be either obtained from the output voltage of the integrator in the PLL scaled with the FM deviation setting of the VCO, or measured directly with a microwave counter. The peak power of the pulsed microwave signals is $-50 \mathrm{dBm}$ (about $-66 \mathrm{dBm}$ in average) at the input end of the semirigid cable.

Figure 2 (b) displays low-field results for both directions of $B$. Besides the apparent Shubinkov-de-Hass oscillations, we can observe additional intriguing features for $B$ below $0.2 \mathrm{~T}$. For frequency higher than few $\mathrm{GHz}$, the high- $B$ data exhibit behaviors similar to a 2DES, showing IQH states. The SdH oscillations become less pronounced at lower $f$ and even completely disappear below $600 \mathrm{MHz}$. In Fig. 2 (c), the data are shown up to $B=11 \mathrm{~T}$. An extra adsorption peak appears at $B=6 \mathrm{~T}$ associated with a unique phase change nearby for $f=255$ $\mathrm{MHz}$, and moves to lower $B$ for higher $f$, indicating that 

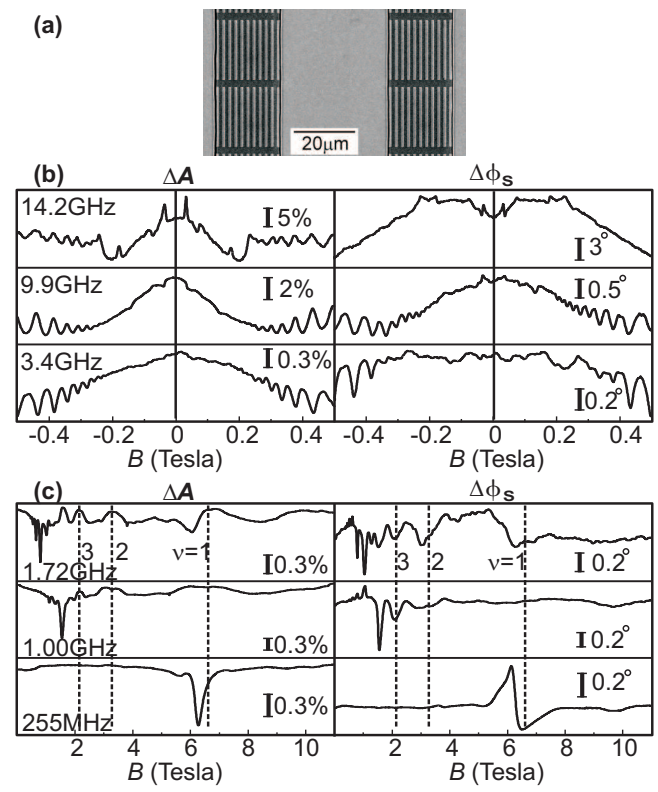

FIG. 2: (a) Simplified schematic diagram for phase detection using a PLL. (b) Block diagram of the vector detection system. The meandering CPW containing a LDES in the gaps is part of the microwave signal path in the PLL.

this feature relates to the edge magnetoplasma ${ }^{12}$ (EMP) excitations. More detailed data and explanation of these interesting results will be published separately. Here we will emphasize mainly the resolution of the measurements and also the polarization of each QW that can be extracted from our data.

The background noise of the data shown in Fig. 2 (b) and (c) is extremely low. The amplitude and phase fluctuations in $\Delta A$ and $\Delta \phi_{s}$ data are less than $0.003 \%$ and $0.001^{\circ}$ for $f \lesssim 10 \mathrm{GHz}$, and $0.05 \%$ and $0.03^{\circ}$ for $f \gtrsim 10 \mathrm{GHz}$, respectively. These resolution limits actually depend on the power reaching the LNA, which is $f$ dependent due to the loss of the sample and the semirigid cables. The average power into the sample and into the LNA are about $-70 \mathrm{dBm}$ and $-76 \mathrm{dBm}$ at $f \sim 1 \mathrm{GHz}$, and down to $-80 \mathrm{dBm}$ and $-107 \mathrm{dBm}$ at $14 \mathrm{GHz}$, respectively. Moreover, the 0.3 $\%$ scale bar in the $\Delta A$ plot is equivalent to only $53 \mathrm{nS} 2 \mathrm{D}$ conductivity in average, which is very small compared to the signal levels in previous studies $\frac{1,2.3 .4 .5 .6}{.1}$

The susceptibility $\gamma$ of each QW and $\Delta \phi_{s}$ can be related 13 by $\gamma=\Delta \phi_{s} \xi^{2} / N \omega Z_{0}$, where $N$ is the total number of QW's, $Z_{0}$ the characteristic impedance of the $\mathrm{CPW}$, and $\xi$ a length scale related to the distribution of the tangential electric field $(E)$ on the surface and the geometry of the CPW. The induced dipole moment of each QW segment, $p$, is then $\gamma E$. For our CPW structure, $\xi$ is about $21 \mu \mathrm{m}$. The $\gamma$ value corresponding to the $0.2^{\circ}$ scale bar in Fig. 2(c) for $255 \mathrm{MHz}$ is about $3 \times 10^{-27} \mathrm{~F} / \mathrm{m}^{2}$. For a signal of $-51.5 \mathrm{dBm}$ peak power, we can estimate $p$ accordingly to be about $3 \times 10^{-25} \mathrm{Cm}$, equivalent to about 17 electrons being transferred across a $0.1 \mu \mathrm{m}$ effective QW width, assuming a $0.3 \mu \mathrm{m}$ depletion length near each edge.

Finally, we want to discuss the effect of the cable length and related instrumental considerations. Usually as we increase $L$, the sensitivity in phase is increased according to Eq. 1, and so is the loop gain of the PLL. However, if $L$ is too big, the PLL will have a very small capture range, and the effect of noise and drift in electronic components become serious. In addition, high-frequency signals will suffer a very severe loss.

In conclusion, we have developed and demonstrated a high-sensitivity vector detection system for very lowpower microwave signals used in a CPW broadband sensor. This system is a very powerful tool in studying the dynamic behaviors, including the electric polarizations, of LDES's at low temperatures.

This work was supported by National Science Council of Republic of China.
1 L. W. Engel, D. Shahar, C. Kurdak, and D. C. Tsui, Phys. Rev. Lett. 71, 2638 (1993).

2 C.-C. Li, L.W. Engel, D. Shahar, D.C. Tsui, and M. Shayegan, Phys. Rev. Lett. 79, 1353 (1997).

${ }^{3}$ P. D. Ye, L. W. Engel, D. C. Tsui, R. M. Lewis, L. N. Pfeiffer, and K. West, Phys. Rev. Lett. 89, 176802 (2002).

${ }^{4}$ R. M. Lewis, P. D. Ye, L. W. Engel, D. C. Tsui, L. N. Pfeiffer, and K. W. West, Phys. Rev. Lett. 89, 136804 (2002).

5 Y. Chen, R. M. Lewis, L. W. Engel, D. C. Tsui, P. D. Ye, L. N. Pfeiffer, and K. W. West, Phys. Rev. Lett. 91, 016801 (2003).

${ }^{6}$ P. D. Ye, L. W. Engel, D. C. Tsui, J. A. Simmons, J. R. Wendt, G. A. Vawter, and J. L. Reno, Phys. Rev. B 65, 121305 (2002).

7 F. Hohls, U. Zeitler, and R. J. Haug, Phys. Rev. Lett. 86, 5124 (2001).

${ }^{8}$ R. M. Lewis and J. P. Carini, Phys. Rev. B 64, 073310
(2001).

${ }^{9}$ For the design of PLLs, please see, e.g., Roland E. Best, Phase-Locked Loops, 5th ed. (McGraw-Hill, Inc., 2003).

10 A. Wixforth, J. Scriba, M. Wassermeier, J. P. Kotthaus, G. Weimann, and W. Schlapp, Phys. Rev. B 40, 7874 (1989).

11 SR830 from Stanford Research Systems, Inc., Sunnyvale, CA.

12 I. Grodnensky, D. Heitmann, K. v. Klitzing, K. Ploog, A. Rudenko, and A. Kamaev, Phys. Rev. B 49, 10778 (1994).

13 This equation can be derived simply by equating the energy stored in the field for the electric dipole moment of all the QW's, $N \gamma E^{2} / 2$, and the energy increment due to change of the effective capacitance per unit length, $\Delta C V^{2} / 2$. The length scale $\xi$ can be shown to be $\left[2\left(C_{a} / \epsilon_{0}\right)\left\langle V^{2} / E^{2}\right\rangle\right]^{1 / 2}$, where $C_{a}$ is the capacitance of the CPW if the substrate is replaced by air, and $\left\langle V^{2} / E^{2}\right\rangle$ is the average of square of voltage signal over square of tangential field on the surface across the gap of the CPW. 